\title{
Steal phenomenon in Sturge-Weber syndrome imitating an ictal electroencephalography change in the contralateral hemisphere: report of 2 cases
}

\author{
Chusak Limotai, MD, ${ }^{1}$ Cristina Y. Go, MD, ${ }^{1}$ Shiro Baba, MD, ${ }^{1}$ Kazuo Okanari, MD, ${ }^{1}$ \\ Ayako Ochi, MD, PhD, ${ }^{1}$ James T. Rutka, MD, PhD, FRCSC, ${ }^{2}$ O. Carter Snead III, MD, FRCPC, and \\ Hiroshi Otsubo, MD'
}

Divisions of ${ }^{1}$ Neurology and ${ }^{2}$ Neurosurgery, The Hospital for Sick Children, Toronto, Ontario, Canada

\begin{abstract}
Infants with Sturge-Weber syndrome (SWS) are considered for surgery if they develop seizures and the seizures prove medically refractory. The authors report on 2 infants (15 and 19 months old) with SWS who underwent scalp video electroencephalography (EEG) and subsequent functional hemispherotomy for intractable partial motor seizures due to extensive left hemispheric angiomatosis. They presented with similar interictal and ictal EEG findings. Ictal EEG showed abrupt high-amplitude delta slow waves, without evolution on the contralateral hemisphere before the build-up of ictal EEG changes on the lesional hemisphere. The patients became seizure free after hemispherotomy. The ictal contralateral slow waves were not a sign of an ictal hemisphere and may indicate prominent ischemic changes resulting from a steal phenomenon of hemispheric angiomatosis during seizure.
\end{abstract}

http://thejns.org/doi/abs/10.3171/2014.12.PEDS14238

KEY WORDS ictal contralateral slow waves; hemispherotomy; seizure; blood flow; infant; oncology

$\mathrm{C}$ LinICal characteristics of Sturge-Weber syndrome (SWS) consist of 1) facial port-wine stains, 2) glaucoma, and 3) leptomeningeal angiomatosis. ${ }^{14,16}$ The disorder manifests with seizures, stroke-like episodes, headache, and developmental delay in children. ${ }^{14}$

Electroencephalography (EEG) in SWS has shown ipsilateral background voltage attenuation, polymorphic delta slow waves, and a paucity of interictal epileptiform discharges. ${ }^{5}$ Ictal EEG changes in the affected hemisphere were rarely seen, and contralateral ictal discharges were seen after clinical seizure onset in 1 case report on SWS. ${ }^{3}$

Single-photon emission computed tomography (SPECT) has demonstrated widespread cortical dysfunction beyond the lesion shown on MRI. ${ }^{6}$ Interictal SPECT has shown hypoperfusion representing ischemic regions in the angioma areas. ${ }^{8}$ Ictal SPECT has demonstrated hyperperfusion in the ipsilateral hemisphere. Subtraction ictal SPECT coregistered to MRI (SISCOM) has shown a massive steal phenomenon in affected areas during seizures, leading to a critical ischemic condition in remote brain regions in a pa- tient with SWS. ${ }^{12}$ To date, however, there has been no report of electrophysiological markers of the steal phenomenon.

We describe 2 cases of SWS with extensive hemispheric leptomeningeal angiomatosis, including information on presurgical evaluations and surgeries. High-amplitude delta slow waves occurred in the contralateral hemisphere, at the ictal onset in both patients. We hypothesized that the paradoxical ictal EEG changes in the contralateral hemisphere resulted from the steal phenomenon by ictal hyperperfusion of the epileptogenic angiomatosis in patients with SWS.

\section{Case Reports}

We retrospectively reviewed the EEG database and patient charts at the Hospital for Sick Children for the period from 2005 to 2011. Seven patients with SWS had been admitted for prolonged scalp video EEG (VEEG). Three patients showed interictal and ictal EEG abnormalities. Two of the 3 patients, who had seizures during VEEG,

ABBREVIATIONS AP = anteroposterior; CBF = cerebral blood flow; EEG = electroencephalography; HFF = high-frequency filter; LFF = low-frequency filter; SWS = SturgeWeber syndrome; VEEG = video EEG.

SUBMITTED May 8, 2014. ACCEPTED December 31, 2014.

INCLUDE WHEN CITING Published online May 8, 2015; DOI: 10.3171/2014.12.PEDS14238.

DISCLOSURE The authors report no conflict of interest concerning the materials or methods used in this study or the findings specified in this paper. 
underwent the epilepsy surgery. We decided that the other patient, because only 1 seizure was captured, was not a surgical candidate. The other 4 patients did not show any seizure during their admission. We analyzed the clinical and EEG findings of the 2 patients who underwent epilepsy surgery.

\section{Case 1}

History

This 15-month-old boy had a normal birth history. At 3 months of age, he began to have focal seizures. These seizures consisted of right-sided mouth clonic movements and at times involved the right arm or right leg. They usually occurred in clusters, occasionally up to 20 clusters per month. A few days prior to his VEEG admission at age 15 months, the boy developed hypomotor seizures ${ }^{9}$ noted as staring episodes associated with bilateral eye blinking. Initial treatment with phenobarbital and phenytoin was unsuccessful. The addition of valproic acid and levetiracetam minimally reduced the seizures.

\section{Examinations}

Neurological examination revealed mild weakness of the right-sided extremities. His deep tendon reflexes were symmetrical. Flexor plantar responses were noted on both sides. He had left eye glaucoma and facial angioma on the left V1 and V2 distributions of the trigeminal nerve. Magnetic resonance imaging revealed an extensive leptomeningeal angioma on the left hemisphere with interval progressive cerebral atrophy over the period of 1 year (Fig. 1A). Video EEG captured 9 seizures, which had all started with staring and eye blinking followed by right arm clonic movements. Ictal EEG onset showed mediumamplitude, rhythmic, 2- to 3-Hz delta slow waves, which were subsequently replaced by low- to medium-amplitude, 6- to 7-Hz theta waves over the left posterior temporooccipital region (electrodes T5, O1). Evolutions of ictal EEG in amplitude, frequency, and distribution were noted in the same hemisphere. Late involvement of the left midtemporocentral region (electrodes T3, C3) was noted. One seizure showed high-amplitude, polymorphic, 1- to $1.5-\mathrm{Hz}$ delta slow waves without evolution in frequency, distribution, and amplitude over the contralateral hemisphere 5 seconds before ictal EEG changes over the lesional hemisphere. Six seizures revealed the simultaneous appearance of ictal EEG changes over the lesional hemisphere and similar high-amplitude delta slow waves over the contralateral hemisphere. The remaining 2 seizures showed similar high-amplitude delta slow waves over the contralateral hemisphere occurring 1 and 4 seconds after ictal
A

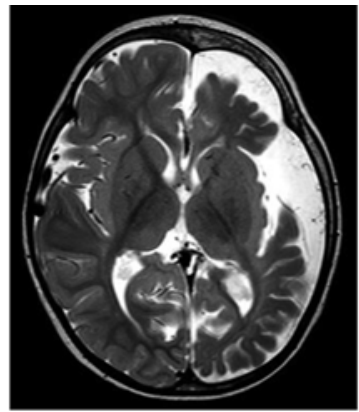

B

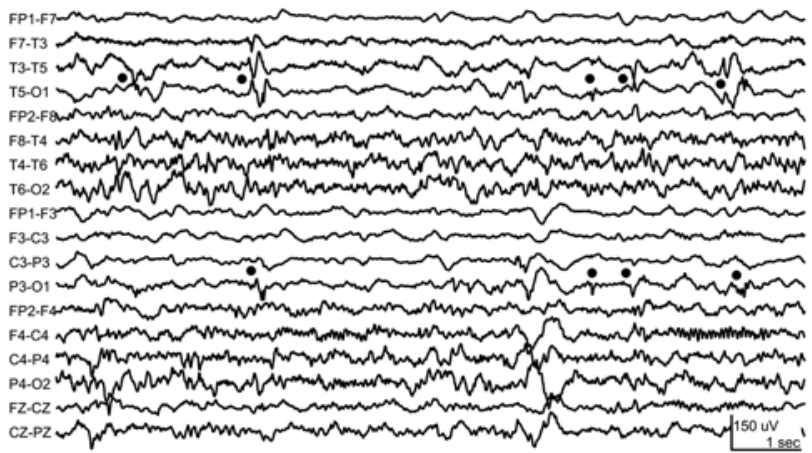

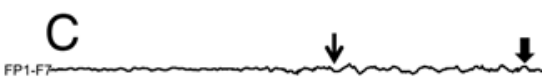

77.Т3

T3-T5 T5-01 FP2-F9 T4-T6 14 6-02 FP1.F3 F3-C3 3 C3.P3 3 P3-01 FP2-F4nm F4-C4

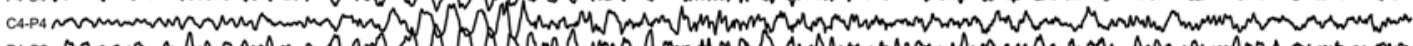

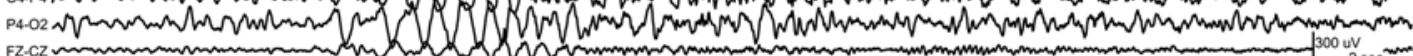
CZ-PZ

FIG. 1. Case 1. A: Axial T2-weighted MR image revealing extensive left hemispheric cerebral atrophy. B: Interictal EEG (anteroposterior [AP] bipolar montage, low-frequency filter [LFF] $1 \mathrm{~Hz}$, high-frequency filter [HFF] $70 \mathrm{~Hz}$ ). Asymmetrical background activity with relatively lower amplitude and decreased faster frequencies were noted over the lesional left hemisphere. Low- to medium-amplitude polyspike or spike and slow waves (closed circles) were identified over the left posterior temporooccipital region (electrodes T5, 01). C: Ictal EEG (AP bipolar montage, LFF $1 \mathrm{~Hz}, \mathrm{HFF} 70 \mathrm{~Hz}$ ). High-amplitude, rhythmic, 1- to 1.5-Hz delta slow waves (thin arrow) start over the contralateral right hemisphere 5 seconds before ictal EEG changes (thick arrow) over the lesional left hemisphere. Over the left hemisphere, medium-amplitude, rhythmic, 2- to 3-Hz delta slow waves were replaced by low- to medium-amplitude, 6- to 7- $\mathrm{Hz}$ theta waves over the left posterior quadrant (electrodes T5, P3, O1) at seizure onset (thick arrow). 
EEG changes over the lesional hemisphere. All seizures lasted 180-200 seconds. Interictally, frequent low- to medium-amplitude polyspike or spike and slow waves were identified over the left posterior temporoparietal regions (electrodes T5, P3). Interictal and ictal EEG findings are shown in Fig. 1B and C and summarized in Table 1.

\section{Surgery and Clinical Outcome}

Left periinsular functional hemispherotomy ${ }^{15}$ was performed on the lesional hemisphere. The patient has been seizure free and no further developmental deterioration has occurred for 8 months since the hemispherotomy. Postoperative follow-up EEG has not been recorded.

\section{Case 2}

History

This 19-month-old girl had a normal birth history and normal development. Focal seizures began when she was 2 months of age. Initially, she had only right arm clonic seizures, occurring on a daily basis. This seizure type had decreased in frequency with an increased dosage of carbamazepine and then disappeared. Hypomotor seizures ${ }^{9}$ associated with staring and unresponsiveness appeared afterward. Antiepileptic drugs administered included phenobarbital and carbamazepine.

\section{Examinations}

Neurological examination revealed hyperreflexia of the right lower extremity and extensor plantar response on the right side. She had neither apparent right-sided weakness nor spasticity. She had left eye glaucoma and a port-wine stain on the left V1 and V2 distributions. Magnetic resonance imaging revealed an extensive leptomeningeal angioma with associated cerebral atrophy on the left hemisphere (Fig. 2A). Video EEG captured 5 seizures, which were all hypomotor seizures. Ictal EEG showed medium-amplitude, rhythmic, 7- to $8-\mathrm{Hz}$ waves on the left mid- to posterior temporal region (electrodes
T3, T5). Evolutions of ictal EEG in amplitude, frequency, and distribution were noted on the same hemisphere. During all 5 seizures, high-amplitude, polymorphic delta slow waves without evolution on the contralateral hemisphere occurred 2-3 seconds before evolving ictal EEG changes on the lesional hemisphere. All seizures lasted 120-150 seconds. Interictally, frequent low- to medium-amplitude polyspike or spike and slow waves were identified over the left posterior quadrant (electrodes T5, P3, O1). Interictal and ictal EEG findings are shown in Fig. $2 \mathrm{~B}$ and $\mathrm{C}$ and summarized in Table 1.

\section{Surgery and Clinical Outcome}

Left periinsular functional hemispherotomy ${ }^{15}$ on the lesional hemisphere was performed. Postoperative CT demonstrated acute swelling of the left hemispheric parenchyma resulting in subfalcine and uncal herniation. A left frontal lobectomy was performed for decompression. The postoperative course was uneventful. Follow-up EEG did not capture any seizure. Interictal discharges over the left posterior quadrant (electrodes T3, P3, O1) disappeared at 2 years after hemispherotomy. The patient had been seizure free and was gaining in developmental status by the 27-month follow-up.

\section{Discussion}

We hypothesize that the paradoxical EEG pattern may represent a steal phenomenon. The paradoxical EEG pattern on the contralateral hemisphere may occur during seizures in patients with extensive hemispheric angiomatosis with significantly compromised cerebral blood flow (CBF).

According to animal studies, a $150 \%-200 \%$ increase in $\mathrm{CBF}$ is required to maintain cortical oxygenation during seizures..$^{10}$ Ictal SPECT and transcranial Doppler studies have disclosed evidence of seizure-related worsening of brain injury in SWS. ${ }^{1}$ An increase in CBF in response to seizures has also been observed in the affected brain re-

\section{TABLE 1. Video EEG findings}

\begin{tabular}{|c|c|c|}
\hline Parameter & Case 1 & Case 2 \\
\hline Interictal EEG & $\begin{array}{l}\text { Low-amplitude polyspikes/spike \& slow waves } \\
\text { at electrodes T5, } 01\end{array}$ & $\begin{array}{l}\text { Low-amplitude polyspike/spike \& slow waves at } \\
\text { electrode T5 > P3, } 01\end{array}$ \\
\hline \multicolumn{3}{|l|}{ Ictal video EEG } \\
\hline Total no. of seizures & 9 & 9 \\
\hline \multicolumn{3}{|l|}{ Clinical findings } \\
\hline Seizure semiology (no. of seizures) & $\begin{array}{l}\text { Hypomotor seizures } \rightarrow \mathrm{rt} \text { arm clonic seizures } \\
\text { (9) }\end{array}$ & $\begin{array}{l}\text { Hypomotor seizures (5), EEG seizures w/o clinical } \\
\text { signs (4) }\end{array}$ \\
\hline Duration (secs) & $180-200$ & 120-150 (hypomotor seizures), 50-60 (EEG seizures) \\
\hline \multicolumn{3}{|l|}{ EEG findings } \\
\hline $\begin{array}{l}\text { Lesional hemisphere: ictal EEG } \\
\text { change }\end{array}$ & $\begin{array}{l}\text { Buildup of delta } \rightarrow \text { theta activity, intermixed } w / \\
\text { beta activity at electrodes } \mathrm{T} 5,01 \rightarrow \text { spread } \\
\text { to electrodes } \mathrm{T3}, \mathrm{C} 3\end{array}$ & $\begin{array}{l}\text { Buildup of theta-alpha intermixed } w / \text { beta activity at } \\
\text { electrode } T 5>T 3,01\end{array}$ \\
\hline $\begin{array}{l}\text { Contralateral hemisphere: delta slow } \\
\text { wave starting at ... (no. of seizures) }\end{array}$ & $\begin{array}{l}5 \text { secs before seizure onset (1) } \\
\text { At seizure onset ( } 6) \\
1-4 \text { secs after seizure onset (2) }\end{array}$ & $\begin{array}{l}\text { 2-3 secs before seizure onset }(5) \\
*\end{array}$ \\
\hline
\end{tabular}

Delta slow wave = high amplitude polymorphic delta slow waves without evolution in frequency, distribution, and amplitude.

* Four EEG seizures showed no significant EEG change in the contralateral hemisphere. 

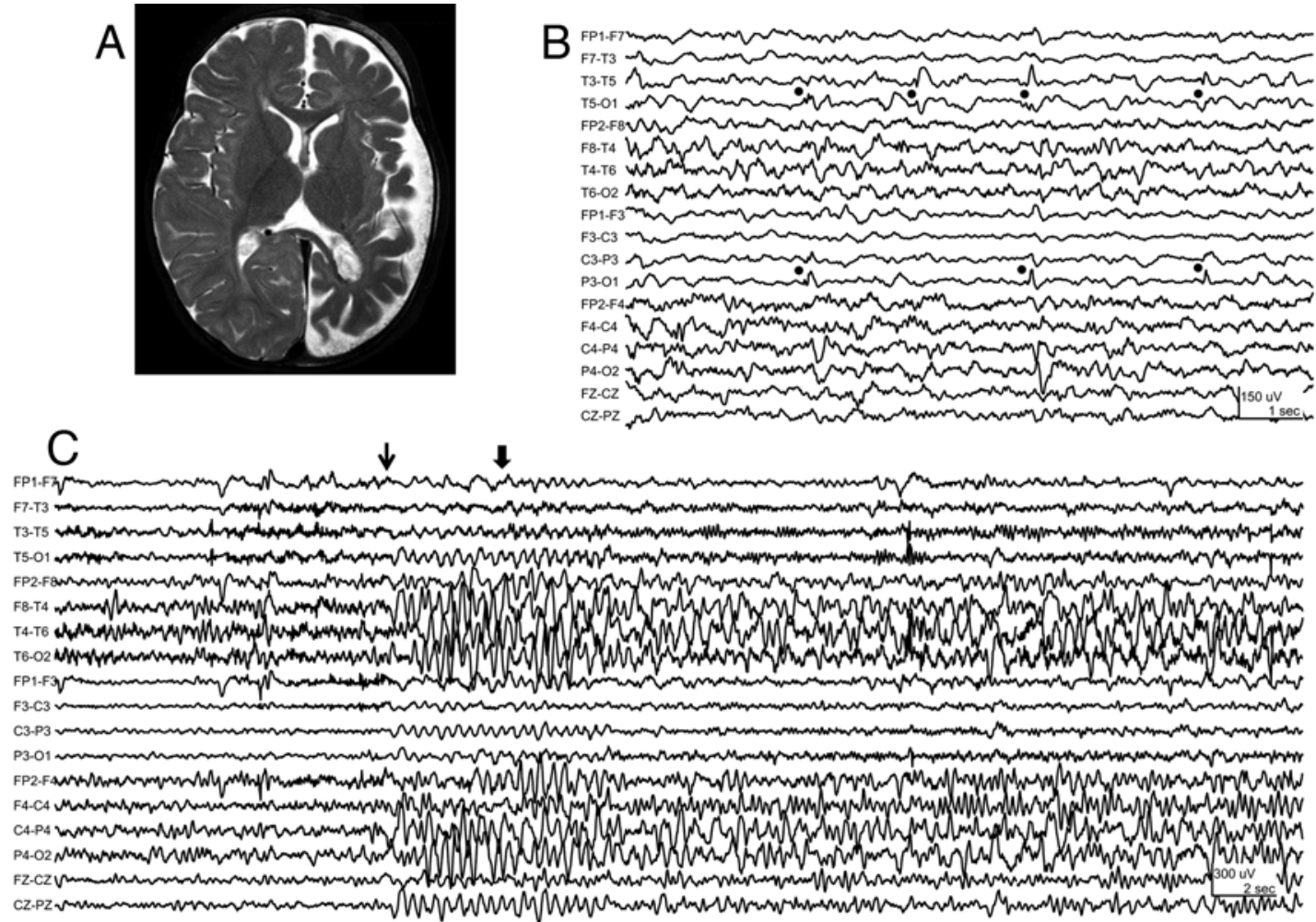

FIG. 2. Case 2. A: Axial T2-weighted MR image revealing extensive left hemispheric cerebral atrophy. B: Interictal EEG (AP bipolar montage, LFF $1 \mathrm{~Hz}, \mathrm{HFF} 70 \mathrm{~Hz}$ ). Asymmetrical background activity with relatively lower amplitude and decreased faster frequencies were noted over the lesional left hemisphere. Low-amplitude polyspike or spike and slow waves (closed circles) were identified over the left posterior quadrant (electrodes T5, P3, 01). C: Ictal EEG (AP bipolar montage, LFF $1 \mathrm{~Hz}, \mathrm{HFF} 70 \mathrm{~Hz}$ ). Highamplitude delta slow waves (thin arrow) start over the contralateral right hemisphere 3 seconds before seizure onset (thick arrow). Low-amplitude beta activities were building up over the left mid- to posterior temporal region (electrodes T3, T5) from seizure onset (thick arrow). Note the contralateral high-amplitude delta slow waves preceding the ictal EEG change over the lesional hemisphere in all 5 clinical seizures.

gion in SWS. Aylett et al. concluded that impaired brain perfusion coupled with the excessive metabolic demand of prolonged seizure activity can result in an exacerbation of ischemic brain injury and significantly contribute to the neurological deterioration in children with SWS. ${ }^{1}$ During seizures in 1 patient included in their report, EEG showed slow-wave activity bilaterally, but the greatest activity was over the left posterior temporal region, which was contralateral to the side of the angioma. ${ }^{1}$ Maria et al. demonstrated progressive changes on both structural and functional imaging studies at both younger (mean age 1.75 years) and older pediatric ages (mean age 15.3 years). ${ }^{11}$ More widespread cortical injury was seen in the older patients. The authors found that developmental delay and stroke-like episodes may be related to progressive bihemispheric dysfunction in SWS. Bilateral hypometabolism appeared to be associated with learning disability even with a unilateral pial angiomatosis. ${ }^{6}$

The mechanism of a steal phenomenon during seizure in SWS may depend on the state of the progressive angioma and dysfunctional cortex with an epileptic disorder. As described by Pinton et al., cortex involved in a vascular malformation was hyperperfused during the 1st year of life before the first seizures. ${ }^{13}$ The classic hypoperfusion appeared after 1 year of age, even in nonepileptic patients. Ictal SPECT has been used to localize ictal hemisphere with surrounding hypoperfusion. ${ }^{7}$ We speculate that after 1 year of age, the vascular malformations in SWS continue to steal blood flow to develop dysfunctional and even epileptic brain. When seizures occur in a subset of patients with SWS, extensive angioma requires massive hyperperfusion and the contralateral hemisphere becomes ischemic.

Bearden and Uthman reported focal polymorphic delta slow waves due to regional cerebral hypoperfusion in patients with cerebral hemodynamic compromise associated with transient ischemic attack. ${ }^{2}$ The continuous polymorphic delta slow waves were highly correlated with a focal structural lesion. Reversible polymorphic delta slow waves could be presented by an acute pathological condition such as seizure and cerebral hypoperfusion. In our cases, the EEG showed prominent contralateral polymorphic slow waves instead of less prominent ictal EEG changes of the atrophic and calcified lesional hemisphere. The repetition rate at the onset of partial seizures ranged from delta to high frequency. Further ictal EEG progression was more likely to occur if a mixed frequency change or frequency increase characterized early evolution. ${ }^{4}$ Thus, the ictal contralateral slow waves without evolution are considered a symptomatic phenomenon of stolen blood flow during seizure due to acute ischemic changes, not ictogenic EEG changes, in SWS. 


\section{Conclusions}

A steal phenomenon associated with a paradoxical EEG finding on the contralateral hemisphere during seizures in a subset of patients with SWS may be a marker of bilaterally impaired neuronal function beyond the lesion. A paradoxical EEG pattern of a high-amplitude slow wave on the contralateral hemisphere should not be interpreted as an ictal EEG onset. Ictal contralateral EEG changes of slow waves are a striking phenomenon of stealing blood flow by hemispheric angiomatosis. One should consider performing surgery to rescue the contralateral hemisphere from the ischemic effect as well as for seizure control.

\section{References}

1. Aylett SE, Neville BG, Cross JH, Boyd S, Chong WK, Kirkham FJ: Sturge-Weber syndrome: cerebral haemodynamics during seizure activity. Dev Med Child Neurol 41:480-485, 1999

2. Bearden S, Uthman B: Cerebral hemodynamic compromise associated with limb shaking TIA and focal EEG slowing. Am J Electroneurodiagn Technol 49:225-243, 2009

3. Bilgin O, Vollmar C, Peraud A, la Fougere C, Beleza P, Noachtar S: Ictal SPECT in Sturge-Weber syndrome. Epilepsy Res 78:240-243, 2008

4. Blume WT, Young GB, Lemieux JF: EEG morphology of partial epileptic seizures. Electroencephalogr Clin Neurophysiol 57:295-302, 1984

5. Brenner RP, Sharbrough FW: Electroencephalographic evaluation in Sturge-Weber syndrome. Neurology 26:629-632, 1976

6. Chugani HT, Mazziotta JC, Phelps ME: Sturge-Weber syndrome: a study of cerebral glucose utilization with positron emission tomography. J Pediatr 114:244-253, 1989

7. Duncan JS: Imaging and epilepsy. Brain 120:339-377, 1997

8. Griffiths PD, Boodram MB, Blaser S, Armstrong D, Gilday DL, Harwood-Nash D: 99mTechnetium HMPAO imaging in children with the Sturge-Weber syndrome: a study of nine cases with CT and MRI correlation. Neuroradiology 39:219-224, 1997

9. Källén K, Wyllie E, Lüders HO, Lachhwani D, Kotagal P: Hypomotor seizures in infants and children. Epilepsia 43:882-888, 2002
10. Kreisman NR, Magee JC, Brizzee BL: Relative hypoperfusion in rat cerebral cortex during recurrent seizures. J Cereb Blood Flow Metab 11:77-87, 1991

11. Maria BL, Neufeld JA, Rosainz LC, Drane WE, Quisling RG, Ben-David K, et al: Central nervous system structure and function in Sturge-Weber syndrome: evidence of neurologic and radiologic progression. J Child Neurol 13:606618, 1998

12. Namer IJ, Battaglia F, Hirsch E, Constantinesco A, Marescaux C: Subtraction ictal SPECT co-registered to MRI (SISCOM) in Sturge-Weber syndrome. Clin Nucl Med 30:39-40, 2005

13. Pinton F, Chiron C, Enjolras O, Motte J, Syrota A, Dulac $\mathrm{O}$ : Early single photon emission computed tomography in Sturge-Weber syndrome. J Neurol Neurosurg Psychiatry 63:616-621, 1997

14. Sturge WA: A case of partial epilepsy, apparently due to a lesion of one of the vasomotor centres of the brain. Transact Clin Soc Lond 12:162-167, 1879

15. Villemure JG, Mascott CR: Peri-insular hemispherotomy: surgical principles and anatomy. Neurosurgery 37:975-981, 1995

16. Weber FP: Right-sided hemi-hypotrophy resulting from right-sided congenital spastic hemiplegia, with a morbid condition of the left side of the brain, revealed by radiograms. J Neurol Psychopathol 3:134-139, 1922

\section{Author Contributions}

Conception and design: Otsubo, Rutka, Snead. Acquisition of data: Otsubo, Limotai, Go, Ochi, Rutka, Snead. Analysis and interpretation of data: Otsubo, Limotai, Go, Baba, Ochi. Drafting the article: Limotai, Baba. Critically revising the article: Otsubo, Baba. Reviewed submitted version of manuscript: Otsubo, Go, Baba, Okanari, Ochi, Rutka, Snead.

\section{Correspondence}

Hiroshi Otsubo, Division of Neurology, The Hospital for Sick Children, 555 University Ave., Toronto, ON M5G 1X8, Canada. email: hiroshi.otsubo@sickkids.ca. 